\title{
Critical role of inhomogeneities in pacing termination of cardiac reentry
}

\author{
Sitabhra Sinha \\ Division of Cardiology, Weill Medical College of Cornell University, New York, New York 10021 \\ and Centre for Condensed Matter Theory, Department of Physics, Indian Institute of Science, \\ Bangalore 560 012, India \\ Kenneth M. Stein \\ Division of Cardiology, Weill Medical College of Cornell University, New York, New York 10021 \\ David J. Christini \\ Division of Cardiology, Weill Medical College of Cornell University, New York, New York 10021 \\ and Department of Physiology and Biophysics, Weill Graduate School of Medical Sciences of Cornell \\ University, New York, New York 10021
}

\begin{abstract}
Reentry around nonconducting ventricular scar tissue, a cause of lethal arrhythmias, is typically treated by rapid electrical stimulation from an implantable cardioverter defibrillator. However, the dynamical mechanisms of termination (success and failure) are poorly understood. To elucidate such mechanisms, we study the dynamics of pacing in one- and two-dimensional models of anatomical reentry. In a crucial realistic difference from previous studies of such systems, we have placed the pacing site away from the reentry circuit. Our model-independent results suggest that with such off-circuit pacing, the existence of inhomogeneity in the reentry circuit is essential for successful termination of tachycardia under certain conditions. Considering the critical role of such inhomogeneities may lead to more effective pacing algorithms.
\end{abstract}

Sudden cardiac death, i.e., death resulting from the sudden abrupt loss of heart function (cardiac arrest) in a person who may or may not have previously diagnosed heart disease, is the leading cause of death in the industrialized world. ${ }^{1}$ It accounts for more than $\mathbf{4 5 0} 000$ deaths in the United States alone each year. ${ }^{2}$ The primary cause of such deaths are ventricular arrhythmias-abnormal rhythms occurring in the lower chambers of the heart due to disorder in the generation and/or propagation of electrical impulses in the heart. The usual method of treatment involves applying a sequence of low-amplitude periodic electrical stimuli locally through an electrode placed on the surface of the cardiac tissue. Such stimulation may often lead to termination of the arrhythmia, but many times it fails to have the desired effect. It has been observed that successful termination is achieved only with stimuli within a particular range of frequencies, which depends on several electrophysiological parameters and therefore varies from subject to subject. In many cases, not only does stimulation fail to terminate arrhythmia, but can in fact be counterproductivetolerated arrhythmias can be driven to more rapid and life-threatening manifestations through stimulation. A detailed understanding of the mechanism underlying the interaction of stimulation with arrhythmia may help to avoid such unfortunate consequences. To this end, we examine the dynamics of stimulus-arrhythmia interactions in 1D and 2D arrhythmia models. Using both mathematical arguments and model simulation results we show that, under certain conditions, successful termination of arrhythmia requires the presence of a zone of inhomogeneity in the arrhythmia generating region.

\section{INTRODUCTION}

Ventricular arrhythmias are often characterized by reentrant waves of excitation. The reentrant wave may propagate around an inexcitable obstacle ("anatomical reentry") $)^{3}$ or may reside in a region of the myocardium that is excitable in its entirety ("functional reentry")., Reentry causes an abnormally rapid heart rate (exceeding 100 beats/min) referred to as tachycardia, which may be fatal when arising in the ventricles because of the corresponding failure to adequately pump blood.

Trains of local electrical stimuli are widely used to restore normal wave propagation in the heart during ventricular tachycardia (VT). Patients at risk for VT are often treated with implantable cardioverter defibrillators (ICD). These devices utilize electrode "leads" that are threaded through the subclavian vein into the heart, where they are typically attached to the endocardial surface of the right ventricle. Via such electrodes, ICDs detect the onset of VT and then attempt to terminate it through rapid low-amplitude electrical stimulation (antitachycardia pacing). However, VT termination algorithms using rapid pacing through an ICD were developed over 20 years ago. These pacing algorithms (such as burst pacing and ramp pacing, explained in Sec. IV) were devised on heuristic principles that do not account for the spatio-temporal dynamics of interacting electrical waves in the heart. Furthermore, the underlying mechanisms govern- 
ing the success and failure of antitachycardia pacing algorithms are not yet clear. Understanding these mechanisms is essential, especially given that such pacing is not always successful and may inadvertently cause tolerated tachycardias to degenerate to more rapid and threatening tachyarrhythmias. A better knowledge of the processes involved in the suppression of VT through such pacing might aid in the design of more effective therapies.

Several factors influence the ability of rapid pacing to interact with VT. The most prominent $\operatorname{are}^{6}$ (a) VT rate (for anatomical reentry this is determined by the length of the VT circuit and conduction velocity around the obstacle), (b) the refractory period (i.e., the duration of time following excitation during which cardiac tissue cannot be re-excited) at the pacing site and in the VT circuit, (c) the conduction time from the pacing site to the VT circuit, and (d) the length of the excitable gap (the region of excitable tissue in the VT circuit between the front and refractory tail of the reentrant wave $\left.^{7}\right)$. Because there are a number of conditions to be satisfied before reentry is successfully terminated, a single stimulus is rarely sufficient. Multiple stimuli are often used, where the earlier stimuli are believed to "peel back" refractoriness to allow the subsequent stimuli to enter the circuit earlier than was possible with only a single stimulus.

Several studies have examined the dynamics of pacing directly on a one-dimensional ring containing a reentrant impulse. ${ }^{4,8-13}$ Most of these studies have been exclusively concerned with an entirely homogeneous ring of cardiac cells. The principal mechanism for termination of reentry in such a geometry (which is effectively that of the reentry circuit immediately surrounding an anatomical obstacle) is unidirectional block. Termination by this process occurs in the following manner. Each stimulus splits into two branches that travel in opposite directions around the reentry circuit. The retrograde branch (proceeding opposite to the direction of the existing reentrant wave) ultimately collides with the reentrant wave, causing mutual annihilation. The anterograde branch (proceeding in the same direction as the reentrant wave) can, depending on the timing of the stimulation, lead to resetting, where the anterograde wave becomes a new reentrant wave, or termination of reentry, where the anterograde wave is blocked by the refractory tail of the original reentrant wave. From continuity arguments, it can be shown that there exists a range of stimuli phases and amplitudes that leads to successful reentry termination..$^{9,10}$ This argument, although later applied to other geometries, ${ }^{12}$ was originally developed for a 1D ring, with the underlying assumption that the pacing site is on the reentry circuit itself. In reality, however, it is unlikely that the pacing site will be so fortuitously located because circuit location is typically not known when a defibrillating device is implanted.

In this study, we examine the dynamics of pacing from a site some distance away from the reentry circuit. Such offcircuit pacing introduces the realistic propagation of the stimulus from the pacing site to the reentry circuit. Because reentrant waves propagate outwardly from, in addition to around, the circuit, the stimulus will be annihilated before it reaches the circuit under most circumstances. Successful propagation to the reentry circuit typically requires utilizing

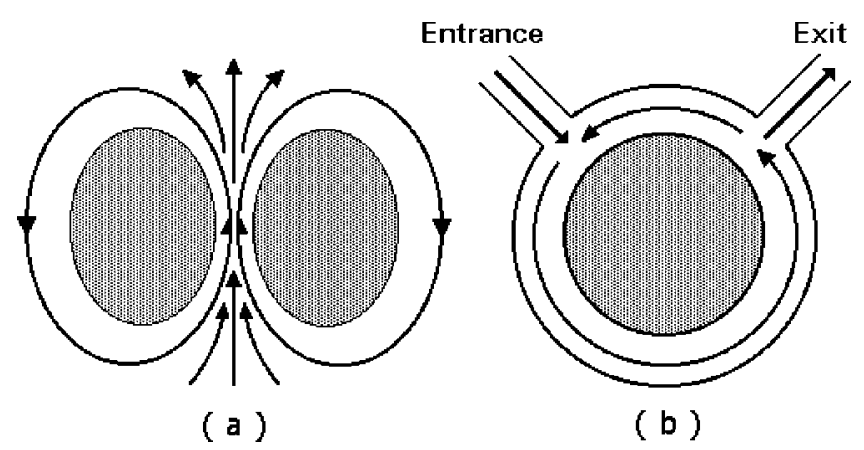

FIG. 1. (a) Schematic diagram of "figure-of-eight" reentry around two anatomical obstacles (shaded regions represent the ischemic central zone). Normal tissue and ischemic border zone are not differentiated in this figure. The arrows indicate the direction of the two counterpropagating rotating waves. (b) One-half of the spatial arrangement involved in figure-of-eight reentry is used to set up a quasi-1D analog model. This abstract model of anatomical reentry has an entrance and an exit sidebranch to signify the region of cardiac myocardium surrounding the circuit.

multiple stimuli to "peel back" refractory tissue incrementally until one successfully arrives at the circuit. ${ }^{6}$ Further, once a stimulus does reach the circuit, its anterograde branch must be blocked by the refractory tail of the reentrant wave, otherwise resetting will occur and termination will fail.

Our results indicate that, for a homogeneous medium, termination of reentry through the above process will be unsuccessful, under certain conditions, if the pacing site is not located on the reentry circuit itself. Thus, inhomogeneities sometime play a critical role in successful termination of reentry in a realistic pacing arrangement. We have examined these issues in a previous study. ${ }^{14}$ Here we look at them in greater detail and extend our results to a $2 \mathrm{D}$ model. The mechanism of the termination process and its failure in a completely homogeneous medium is analyzed in detail in Sec. III, preceded by a short description of the type of anatomical reentry we have examined in Sec. II. Section III also contains the results of our numerical simulations of onedimensional models. In Sec. IV, the results of our twodimensional model simulations are described, while Sec. V contains a short exposition of our conclusions.

\section{FIGURE-OF-EIGHT REENTRY}

The underlying substrate for anatomical reentry in the ventricles is typically scar tissue that serves as anatomical conduction barriers. This is often the result of myocardialinfarction induced ischemia, i.e., oxygen deprivation of cardiac tissue due to obstruction of the coronary artery supplying oxygenated blood to that area. Experimental studies on patterns of activation during the acute phase of myocardial ischemia have shown reentrant activity around and across the ischemic border zone (e.g., in tissue which has healed after undergoing an episode of myocardial infarction ${ }^{15}$ ). This type of activity often takes the form of "figure-of-eight reentry," which consists of two adjacent counter-rotating reentry circuits that share a common pathway. Propagation in these circuits occurs within normal tissue and the ischemic border zone, while the anatomical barriers are the scar tissue comprising the ischemic central zone [Fig. 1(a)]. This situation 
may be fatal if the VT rate is too rapid or if, as occurs in a large number of cases, the VT degenerates into ventricular fibrillation. ${ }^{16}$

For our study of pacing termination of reentry, we looked specifically at a geometry of anatomical obstacles which give rise to a figure-of-eight pattern. For the 2D model we explicitly modeled this by using two rectangular inexcitable patches ("scar tissue") at the center of the simulation domain with a narrow channel for wave propagation between them. The pacing site is placed away from the immediate vicinity of the patches, as we are investigating off-circuit pacing (i.e., pacing from a location at some distance from the reentry circuit). For the 1D model we consider one-half of this geometry, whereby we consider the reentry circuit around one of these inexcitable regions, with an entrance and an exit sidebranch [Fig. 1(b)] (the symmetry of the 2D spatial arrangement makes this a reasonable simplification). Because the pacing site is located away from the circuit, we consider it to be situated at a point in the entrance sidebranch.

Real scar tissue is unlikely to have regular shapes like squares or circles-rather they will have irregular contours. However, if one considers only the portion of the reentry circuit immediately surrounding the inexcitable region, then this can be modeled by a smooth circuit such as a circle. The 1D model represents this kind of circuit, which does not have any sharp edges. To ensure that the introduction of angular extremities do not change the qualitative picture, we look at scars in the shape of squares in the 2D model simulations. The presence of sharp edges in the square patches of inexcitable tissue did not effect our qualitative results. This implies that our results may be valid even if the inexcitable regions have realistic irregular contours.

\section{ONE-DIMENSIONAL MODEL}

Let us consider a reentrant circuit as a 1D ring of length $L$ with separate entrance and exit sidebranches (Fig. 2). This arrangement is an abstraction of the spatial geometry involved in anatomical reentry. ${ }^{6}$ We place the pacing site on the entrance sidebranch at a distance $z$ from the circuit to observe whether reentry is terminated by a mechanism akin to a unidirectional block. We use the entrance sidebranch as the point of spatial origin $(x=0)$ to define the location of the wave on the ring. The conduction velocity and refractory period at a location a distance $x$ away (in the clockwise direction) from the origin are denoted by $c(x)$ and $r(x)$, respectively.

For a homogeneous medium, $c(x)=c, r(x)=r(c, r$ are constants). Therefore, the length of the region in the ring which is refractory at a given instant is $l=c r$. For sustained reentry to occur, an excitable gap must exist (i.e., $L>c r$ ). We consider the circuit length $L$ to be larger than the critical value required for periodic reentry (as opposed to, e.g., quasiperiodic regimes for shorter values of $L$ ), such that the conduction velocity and refractory period are constant. We assume that restitution effects, i.e., the variation of the action potential duration (APD) as a function of the recovery time, can be neglected. For convenience, we associate $t=0$ with

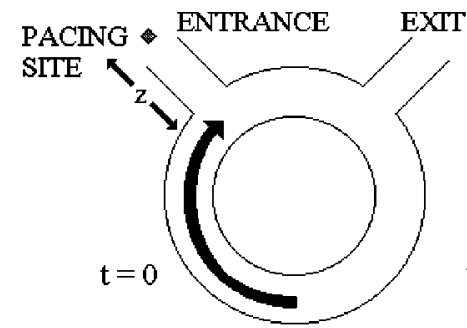

(a)

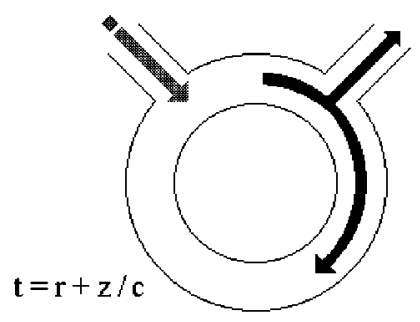

(c)

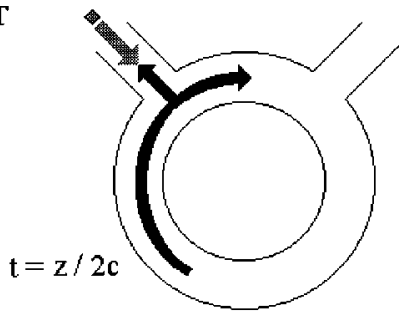

(b)

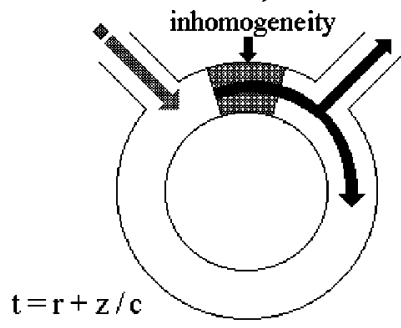

(d)
FIG. 2. Schematic diagram of reentry in a $1 \mathrm{D}$ ring illustrating the necessity of a region of inhomogeneity for successful termination of reentry by pacing. At $t=0$ the reentrant wave reaches the entrance sidebranch (a). At $t$ $=z / 2 c$ the reentrant wave propagating through the sidebranch and the first stimulus mutually annihilate each other (b). At $t=r+(z / c)$ the second stimulus reaches the reentry circuit by which time the refractory tail is a distance $z$ away from the sidebranch (c). The presence of a region of inhomogeneity proximal to the pacing site makes it possible that the anterograde branch of the second stimulus will encounter a refractory region behind the reentrant wave (d).

the time when the reentrant wavefront is at $x=0$ (i.e., the entrance sidebranch) [Fig. 2(a)]. Let us assume that a stimulus is applied at $t=0$. This stimulus will collide with the branch of the reentrant wave propagating out through the entrance sidebranch at $t=z / 2 c$ [Fig. 2(b)]. The pacing site will recover at $t=r$ and if another stimulus is applied immediately it will reach the reentry circuit at $t=r+(z / c)$ [Fig. 2(c)]. By this time the refractory tail of the reentrant wave will be at a distance $x=z$ away from the entrance sidebranch and the anterograde branch of the stimulus will not be blocked. Thus, when $z>0$, it is impossible for the stimulus to catch up to the refractory tail in a homogeneous medium. This results in resetting of the reentrant wave rather than its termination.

Note that if the first stimulus is given at a time $t<-z / c$, it reaches the reentry circuit before the arrival of the reentrant wave. As a result, the retrograde branch collides with the oncoming reentrant wave, while the anterograde branch proceeds to become the reset reentrant wave. Even in the very special circumstance that the reentrant wave reaches the entrance sidebranch exactly at the same instant that the stimulus reaches the circuit, the collision of the two waves lead to a local depolarization that allows the propagation to continue along the reentrant circuit. As a result, pacing termination through a process analogous to a unidirectional block seems all but impossible in a homogeneous reentry circuit under the assumptions outlined above.

The situation changes, however, if an inhomogeneity (e.g., a zone of slow conduction) exists in the circuit [Fig. 2(d)]. Such slow conduction zones have been detected within VT reentry circuits in chronic infarct scar tissue in the 
heart. ${ }^{17,18}$ In this case, the above argument no longer holds because the waves travel faster or slower depending on their location in the circuit. As a result, stimuli may arrive at the circuit from the pacing site and encounter a region that is still refractory. This leads to successful block of the anterograde branch of the stimulus, while the retrograde branch annihilates the reentrant wave (as in the homogeneous case), resulting in successful termination. The success of pacing in this scenario will depend on the location of the inhomogeneity. For example, as the inhomogeneity in Fig. 2(d) is moved in the clockwise direction, termination likelihood decreases because the stimulus will have a longer distance to traverse before reaching the inhomogeneity, which will correspondingly have a longer time in which to recover. This is supported by electrophysiological studies of pacing in cardiac tissue. $^{19}$

Note that the refractory period $r$ refers to the absolute refractory period, i.e., the period immediately following the excitation of the tissue when it cannot be re-excited even with stimulation of arbitrarily high amplitude. One can additionally consider a relative refractory period following the absolute refractory period, when the medium is capable of being excited but the threshold stimulus required to excite it is increased beyond that required normally. This does not change the arguments introduced above. If the stimulus enters the circuit during the relative refractory period, it will propagate slower than the reentrant wave ( $c$ is effectively smaller in the relative refractory tissue) and the stimulus is even less likely to catch up with the refractory tail of the reentrant wave. Therefore, termination in the absence of inhomogeneity in the reentry circuit appears to be impossible even if one considers a relative refractory period, in addition to an absolute refractory period.

Similar arguments may apply for other types of inhomogeneity. For example, existence of a region having longer refractory period will lead to the development of patches of refractory zones in the wake of the reentrant wave. If the anterograde branch of the stimulus arrives at such a zone before it has fully recovered, it will be blocked. ${ }^{3}$

The conclusion that the existence of inhomogeneity in the reentry circuit is essential, under certain conditions, for pacing termination of VT by a mechanism similar to unidirectional block, is also supported by the simulation results reported in the following two subsections.

\section{A. Luo-Rudy simulation results}

We assume that the cardiac impulse propagates in a continuous one-dimensional ring of tissue (ignoring the microscopic cell structure) with ring length $L$, representing a closed pathway of circus movement around an anatomical obstacle (e.g., scar tissue). The propagation is described by the partial differential equation,

$$
\partial V / \partial t=I_{\text {ion }} / C_{m}+D \nabla^{2} V
$$

where $V(\mathrm{mV})$ is the membrane potential, $C_{m}=1 \mu \mathrm{F} \mathrm{cm}^{-2}$ is the membrane capacitance, $D\left(\mathrm{~cm}^{2} \mathrm{~s}^{-1}\right)$ is the diffusion constant, and $I_{\text {ion }}\left(\mu \mathrm{A} \mathrm{cm}^{-2}\right)$ is the cellular transmembrane ionic current density. We used the Luo-Rudy I action potential model, ${ }^{20}$ in which $I_{\text {ion }}=I_{\mathrm{Na}}+I_{\mathrm{si}}+I_{\mathrm{K}}+I_{\mathrm{K} 1}+I_{\mathrm{Kp}}+I_{b} \cdot I_{\mathrm{Na}}$
$=G_{\mathrm{Na}} m^{3} h j\left(V-E_{\mathrm{Na}}\right)$ is the fast inward $\mathrm{Na}^{+}$current, $I_{\mathrm{si}}$ $=G_{\mathrm{si}} d f\left(V-E_{\mathrm{si}}\right)$ is the slow inward current, $I_{\mathrm{K}}=G_{\mathrm{K}} x x_{i}(V$ $\left.-E_{\mathrm{K}}\right)$ is the slow outward time-dependent $\mathrm{K}^{+}$current, $I_{\mathrm{K} 1}$ $=G_{\mathrm{K} 1} K 1_{\infty}\left(V-E_{\mathrm{K} 1}\right)$ is the time-independent $\mathrm{K}^{+}$current, $I_{\mathrm{Kp}}=0.0183 K_{p}\left(V-E_{\mathrm{Kp}}\right)$ is the plateau $\mathrm{K}^{+}$current, and $I_{b}$ $=0.03921(V+59.87)$ is the total background current. $m, h$, $j, d, f, x$, and $x_{i}$ are the gating variables satisfying differential equations of the type, $d y / d t=\left(y_{\infty}-y\right) / \tau_{y}$, where $y_{\infty}$ and $\tau_{y}$ are dimensionless quantities which are functions solely of $V$. The external $\mathrm{K}^{+}$concentration is set to be $[\mathrm{K}]_{0}=5.4 \mathrm{mM}$, while the intracellular $\mathrm{Ca}^{2+}$ concentration obeys $d[\mathrm{Ca}]_{i} / d t=-10^{-4} I_{\mathrm{si}}+0.07\left(10^{-4}-[\mathrm{Ca}]_{i}\right)$. The details of the expressions and the values used for the constants can be found in Ref. 20. We solve the model by using a forward-Euler integration scheme. We discretize the system on a grid of points in space with spacing $\delta x=0.01 \mathrm{~cm}$ and use the standard three-point difference stencil for the 1D Laplacian. The spatial grid consists of a linear lattice with $N$ points; in this study we have used values of $N$ ranging from 500 to 2500 . The time step is $\delta t=0.005 \mathrm{~ms}$. Stimulation is through a square pulse of current with amplitude 3 times the threshold at rest, applied over a length $300 \mu \mathrm{m}$ for a duration of $0.04 \mathrm{~ms}$.

The initial condition is a stimulated wave at some point in the medium with transient conduction block on one side to permit wavefront propagation in a single direction only. The conduction block is realized by applying the initiating impulse at the refractory end of a preceding wave. This initiates a reentrant wave which roughly corresponds to an anatomically-mediated tachycardia. Extrastimuli are then introduced from a pacing site.

To understand the process of inhomogeneity-mediated termination, we introduced a zone of slow conduction in the ring. The slow conduction observed in cardiac tissue under experimental ischemic conditions (lack of oxygen to the tissue $^{21}$ can be reasonably attributed to a high degree of cellular uncoupling, as demonstrated by model simulations. ${ }^{22}$ In our model, slow conduction was implemented by varying the diffusion constant $D^{\prime}$ from the value of $D$ used for the remainder of the ring. The length and diffusion constant $\left(D^{\prime}\right)$ of the zone were varied to examine their effect on the propagation of the anterograde branch of the stimulus. The reentrant wave activates the point in the ring (proximal to the zone of slow conduction) chosen to be the origin $(x=0)$, where the stimulus enters the ring, at time $t=T_{0}$. At time $t$ $=T_{1}$, the first stimulus was given at $x=0$ followed by a second stimulus at $t=T_{2}$. The first stimulus is able to terminate the reentry by itself if it is applied when the region on one side of it is still refractory-leading to unidirectional propagation. This is identical to the mechanism studied previously for terminating reentry by pacing within a $1 \mathrm{D}$ ring. ${ }^{9}$ However, as mentioned earlier, we are interested in the effect of pacing from a site away from the reentry circuit. In that case, it is not generally possible for the first stimulus to arrive at the reentry circuit exactly at the refractory end of the reentrant wave (as discussed above). Therefore, we consider the case when the first stimulus is unable to block the anterograde conduction by itself. We have used values of $T_{1}$ for which the first stimulus can give rise to both the anterograde 

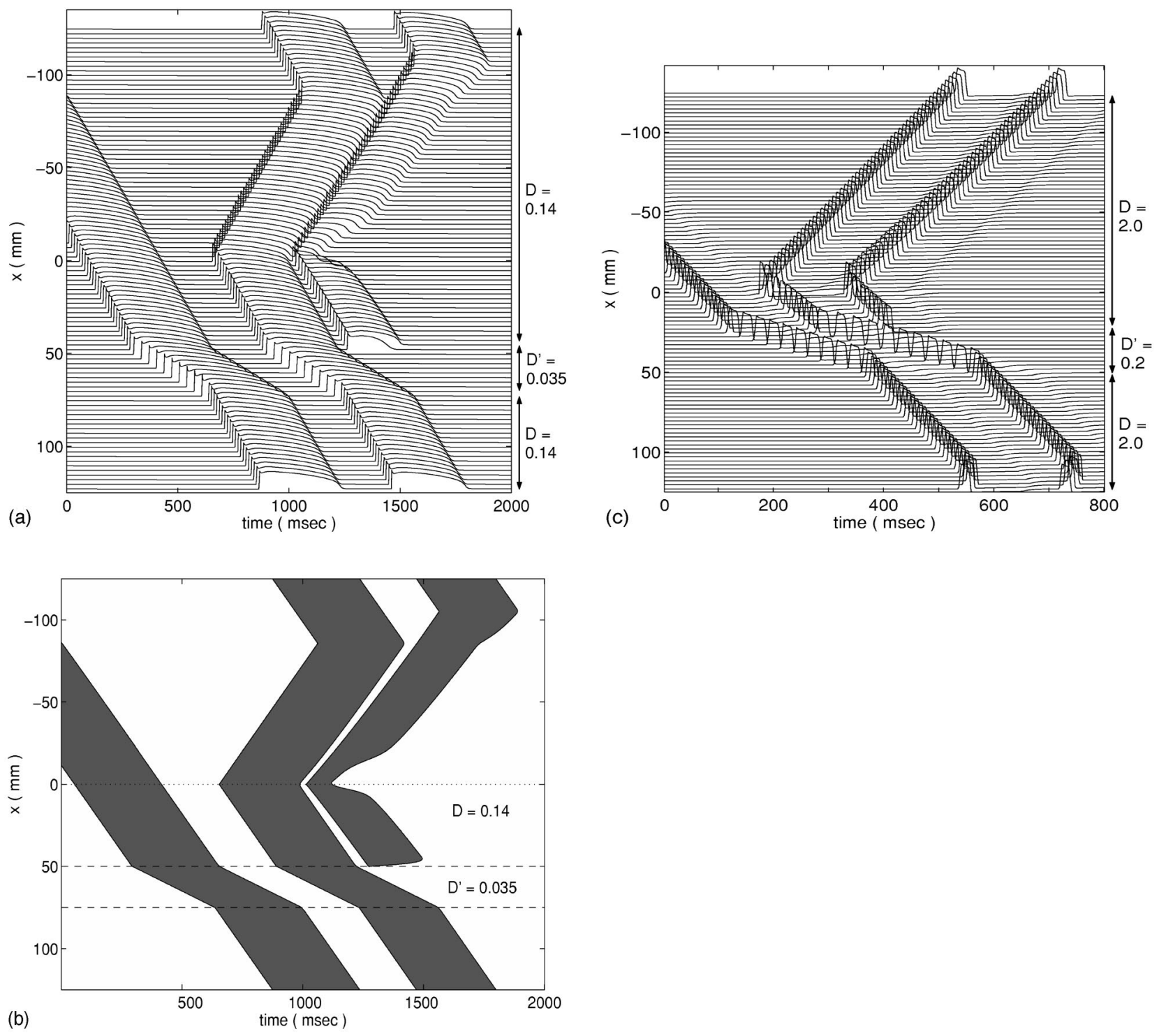

FIG. 3. (a) Plot of the membrane potential showing spatiotemporal propagation of a reentrant wave in a Luo-Rudy ring of length $250 \mathrm{~mm}$, successfully terminated by pacing with two stimuli. The zone of slow conduction is between $x=50 \mathrm{~mm}$ and $x=75 \mathrm{~mm}$. In this region the diffusion constant changes from $D=0.14$ to $D^{\prime}=0.035 \mathrm{~cm}^{2} / \mathrm{s}$ with an infinite gradient at the boundaries. The reentrant wave activates the site at $x=0 \mathrm{~mm}$ at $T_{0}=54.53 \mathrm{~ms}$. The first stimulus is applied at $x=0 \mathrm{~mm}$ at $T_{1}=653.3 \mathrm{~ms}$ (coupling interval $=598.77 \mathrm{~ms}$ ) and the second is applied at $x=0 \mathrm{~mm}$ at $T_{2}=1013.3 \mathrm{~ms}$ (pacing interval=360 ms). (b) Same figure as above but showing the spatial profile of the APD (in gray) associated with the reentrant wave and the activation fronts produced by the pair of applied stimuli. APD is measured as the time interval between successive crossings of $-60 \mathrm{mV}$ by the membrane potential $V$. (c) Plot of the excitability variable $e$ showing spatiotemporal propagation of a reentrant wave in a Panfilov ring of length $250 \mathrm{~mm}$, successfully terminated by pacing with two stimuli. The zone of slow conduction is between $x=25 \mathrm{~mm}$ and $x=50 \mathrm{~mm}$. In this region the diffusion constant changes from $D=2.0$ to $D^{\prime}=0.2 \mathrm{~cm} / \mathrm{s}$ with an infinite gradient at the boundaries. The reentrant wave activates the site at $x=0 \mathrm{~mm}$ at $T_{0}=39.71 \mathrm{~ms}$. The first stimulus is applied at $x=0 \mathrm{~mm}$ at $T_{1}$ $=172.0 \mathrm{~ms}$ (coupling interval $=132.29 \mathrm{~ms}$ ) and the second is applied at $x=0 \mathrm{~mm}$ at $T_{2}=327.0 \mathrm{~ms}$ (pacing interval $=155.0 \mathrm{~ms}$ ).

as well as the retrograde branches. Figure 3(a) shows an instance of successful termination of the reentrant wave in a ring of length $L=250 \mathrm{~mm}$, where the anterograde branch of the second stimulus is blocked in the region of slow conduction (at time $t \sim 1275 \mathrm{~ms}, x=50 \mathrm{~mm}$ ). Figure 3(b) shows the spatial profile of the APDs for the reentrant wave and the activation fronts produced by the pair of stimuli at times $T_{1}$ and $T_{2}$. The APD of an activation wave is measured as the time-interval between successive crossing of $-60 \mathrm{mV}$ by the transmembrane potential $V$.

Different values of coupling interval $\left(\mathrm{CI}=T_{1}-T_{0}\right)$ and pacing interval $\left(\mathrm{PI}=T_{2}-T_{1}\right)$ were used to find which pa- rameters led to block of the anterograde wave. Figure 4(a) is a parameter space diagram which shows the different parameter regimes where termination was achieved. The data shown are for a zone of slow conduction in a ring of length $250 \mathrm{~mm}$, where the zone of slow conduction is between $x$ $=50 \mathrm{~mm}$ and $x=75 \mathrm{~mm}$. The diffusion constant changes sharply from $D=0.14$ to $D^{\prime}=0.035 \mathrm{~cm}^{2} / \mathrm{s}$ at the boundary of the inhomogeneity, with an infinite gradient. We also observed reentry termination when the diffusion constant changes gradually at the boundaries, i.e., with a finite gradient. For instance, when the diffusion constant changes from $D=0.22$ to $D^{\prime}=0.032 \mathrm{~cm}^{2} / \mathrm{s}$ at the boundary with a gradient 

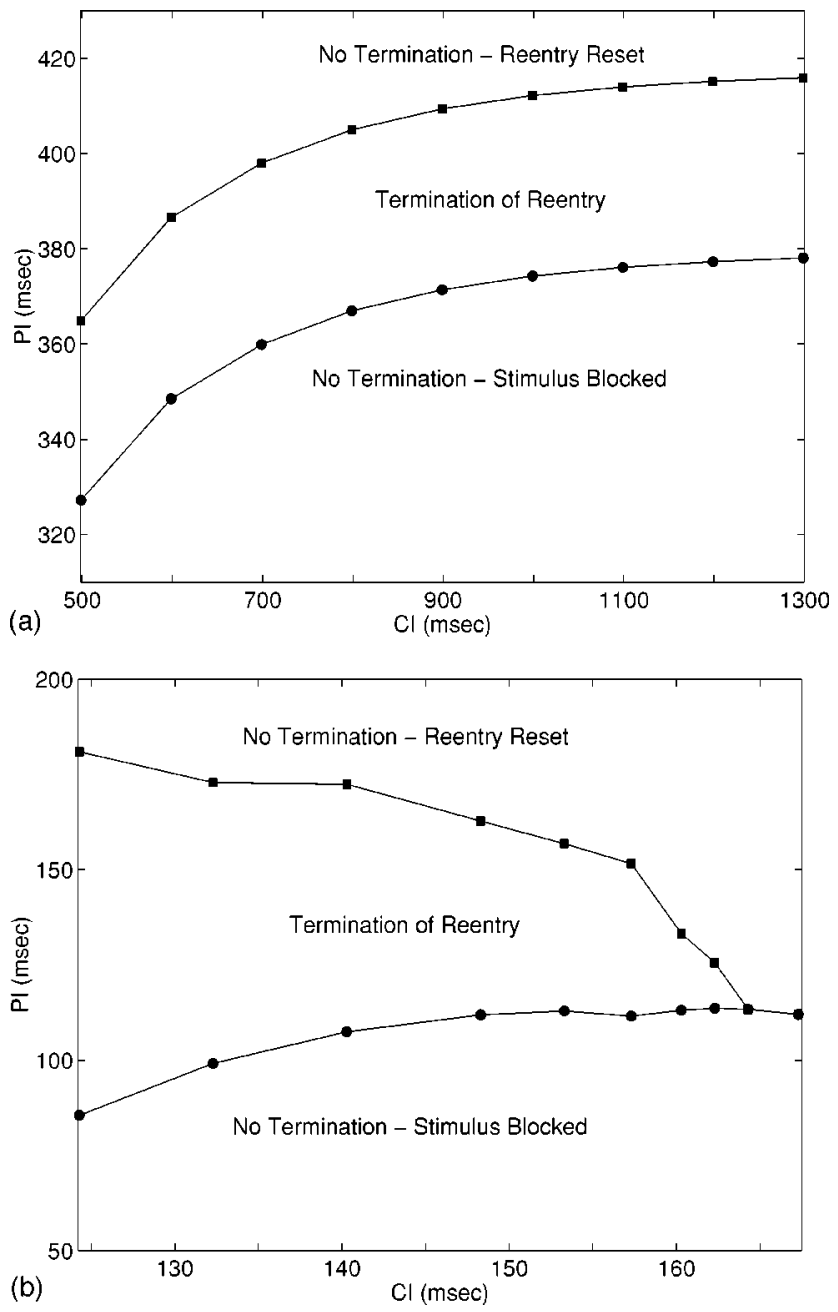

FIG. 4. (a) Parameter space diagram of coupling interval (CI) and pacing interval (PI) at which termination occurs in the 1D Luo-Rudy ring of length $250 \mathrm{~mm}$ with a zone of slow conduction $25 \mathrm{~mm}$ long. The VT period around the ring is $1414.72 \mathrm{~ms}$. The line connecting the circles represents the critical pacing interval value below which the second stimulus gets blocked by the refractory tail of the first stimulus. The lines connecting the squares represent the pacing interval below which the second stimulus is blocked in the anterograde direction in the zone of slow conduction (leading to successful termination) for boundaries between regions with $D=0.14$ and $D^{\prime}$ $=0.035 \mathrm{~cm}^{2} / \mathrm{s}$ having an infinite gradient. For longer PI, the second stimulus propagates through the inhomogeneity and only resets the reentrant wave. Note that, the APD of the reentrant wave at the point of stimulation $(x=0)$ is $359.15 \mathrm{~ms}$. (b) Parameter space diagram of coupling interval (CI) and pacing interval (PI) at which termination occurs in the 1D Panfilov ring of length $250 \mathrm{~mm}$ with a zone of slow conduction $25 \mathrm{~mm}$ long. The VT period around the ring is $883.08 \mathrm{~ms}$. The line connecting the circles represent the pacing intervals below which the second extrastimulus gets blocked by the refractory tail of the first extrastimulus. The lines connecting the squares represent the pacing intervals below which the second extrastimulus is blocked in the anterograde direction at the edge of the region of slow conduction (leading to successful termination) for boundaries between regions with $D=2.0$ and $D^{\prime}=0.2 \mathrm{~cm}^{2} / \mathrm{s}$ having an infinite gradient. For pacing intervals higher than these values, the second extrastimulus propagates bidirectionally and only resets the reentrant wave (i.e., termination fails).

of $\Delta D / \Delta x=3.8 \mathrm{~cm} / \mathrm{s}$, then termination is observed to occur between $\mathrm{PI}=350.3-371.3 \mathrm{~ms}$, for $\mathrm{CI}=612.4 \mathrm{~ms}$. Our results indicate that a smaller gradient at the boundary between the two regions sharply decreases the range of pacing intervals (for a fixed coupling interval) which lead to successful termination. In fact, if the gradient becomes small enough, no reentry termination is observed at any pacing parameter (CIPI) value. This suggests that a sufficiently sharp transition between regions of fast and slow conduction is required for the inhomogeneity to disrupt conduction dynamics enough to lead to pacing termination. The effect of sharp versus gradual transition in intercellular resistance on the propagation of activation fronts in a 1D cable has been observed before in Refs. 23, where it was found that a sharp rise in resistance is more detrimental to wave propagation than a gradual increase, supporting our observations. However, whereas the previous studies reported that propagation block occurred as the wavefront was entering the low resistance region from the high resistance region, we observe the block to occur as the wave entered the high resistance region from the low resistance region. This indicates that the conduction block reported here is a dynamical effect linked to the local prolongation of the APD at the boundary of the zone of slow conduction $^{14}$ and therefore, is very different from that reported in the previous studies where the block was linked to a process of local activation.

We found that varying the size of the zone of slow conductance (specifically, between $1.5 \mathrm{~mm}$ and $25 \mathrm{~mm}$ ) had no qualitative effect on the results. This is likely due to the fact that the conduction disruption that causes termination occurs in the inhomogeneity's boundary region. We also observed that increasing the distance of the zone of slow conduction from the stimulation site decreases the range of pacing intervals which lead to successful reentry termination for a fixed coupling interval. For instance, for $\mathrm{CI}=598.77 \mathrm{~ms}$, displacing the inhomogeneity by $30 \mathrm{~mm}(12 \%)$ away from the site of stimulation, decreased by $5.6 \mathrm{~ms}(\sim 15 \%)$ the range of pacing intervals for which reentry termination occurs.

\section{B. Fitzhugh-Nagumo simulation results}

To ensure that these results were not model dependent, especially on the details of ionic currents, we also looked at a modified Fitzhugh-Nagumo-type excitable media model of ventricular activation proposed by Panfilov. ${ }^{24}$ This model is defined by the two equations governing the excitability $e$ and recovery $g$ variables,

$$
\begin{aligned}
& \partial e / \partial t=D \nabla^{2} e-f(e)-g, \\
& \partial g / \partial t=\epsilon(e, g)(k e-g) .
\end{aligned}
$$

The function $f(e)$, which specifies fast processes (e.g., the initiation of the action potential) is piecewise linear: $f(e)$ $=C_{1} e$, for $e<e_{1}, f(e)=-C_{2} e+a$, for $e_{1} \leqslant e \leqslant e_{2}$, and $f(e)=C_{3}(e-1)$, for $e>e_{2}$. The function $\epsilon(e, g)$, which determines the dynamics of the recovery variable, is $\epsilon(e, g)$ $=\epsilon_{1}$ for $e<e_{2}, \epsilon(e, g)=\epsilon_{2}$ for $e>e_{2}$, and $\epsilon(e, g)=\epsilon_{3}$ for $e<e_{1}$ and $g<g_{1}$. We use the physically appropriate parameter values given in Ref. 25.

We solve the model by using a forward-Euler integration scheme. The system is discretized on a grid of points with spacing $\delta x=0.05 \mathrm{~cm}$ and the standard three-point difference stencil is used for the 1D Laplacian. The spatial grid consists of a linear lattice with $N$ points; in this study we have used values of $N$ ranging from 500 to 1000 . The time step is $\delta t$ $=0.11 \mathrm{~ms}$. Decreasing the space step by a factor of 2 and the 
time step by a factor of 4 , changed the conduction velocity by $<5 \%$. The scaled diffusion constant for the homogeneous ring is $D=2 \mathrm{~cm}^{2} / \mathrm{s}$. Stimulation is through a square pulse of current with amplitude 4 times the threshold at rest, applied over a length $0.15 \mathrm{~cm}$ for a duration of $7.5 \mathrm{~ms}$.

The initial condition is a stimulated wave at some point in the medium with transient conduction block on one side to permit wavefront propagation in a single direction only. The conduction block is realized by making the corresponding region refractory. Inhomogeneity was introduced into the model through a region of slow conduction with a diffusion constant $D^{\prime}$, different from the value of $D$ used for the remainder of the ring. The length and diffusion constant of the inhomogeneous region were varied to examine their effects on the success of reentry termination.

As in the Luo-Rudy simulation, the point where the stimulus enters the ring is chosen to be the origin $(x=0)$. At time $t=T_{0}$, the reentrant wave activates the origin. The first stimulus is applied at $x=0$ at time $t=T_{1}$ followed by a second stimulus at time $t=T_{2}$. We use only values of coupling interval for which the first stimulus can give rise to both anterograde and retrograde branches. In this scenario, termination occurs by block of the anterograde branch of the second stimulus at the boundary of the inhomogeneity. Figure 3(c) shows an instance of successful termination of the reentrant wave in a ring of length $L=250 \mathrm{~mm}$, for a coupling interval of $132.29 \mathrm{~ms}$ and a pacing interval of $155.0 \mathrm{~ms}$ (at time $t \sim 404.1 \mathrm{~ms}, x=25 \mathrm{~mm}$ ).

This two-variable model lacks any description of ionic currents and does not exhibit either the restitution or dispersion property of cardiac tissue. As expected, such differences resulted in quantitative changes in termination requirements. Specifically, the parameter regions at which termination occurs for the Panfilov model [Fig. 4(b)] are different from those shown in Fig. 4(a) for the Luo-Rudy model. Nevertheless, despite quantitative differences, these fundamentally different models shared the requirement of an inhomogeneity for termination, thereby supporting the model independence of our findings.

\section{TWO-DIMENSIONAL MODEL}

The necessity of inhomogeneity in the reentry circuit extends to the case of pacing termination in a twodimensional model. A few studies have looked at the role of inhomogeneities in such a setting. ${ }^{3,26}$ Reference 3 examined the role of a region of increased refractoriness placed in the reentry circuit, while Ref. 26 investigated the effects of highfrequency stimulation of excitable media with an inexcitable obstacle. Both studies noted conditions in which reentrant patterns are eliminated. However, by building upon the 1D model results of Sec. III and connecting it to 2D models, in this section we more firmly establish (than suggested in earlier studies) the important role of inhomogeneities in pacing termination of reentry.

We solve the Panfilov model (discussed in the previous section) in two dimensions by using a forward-Euler integration scheme. We discretize our system on a grid of points in space with spacing $\delta x=0.5$ dimensionless units and use the

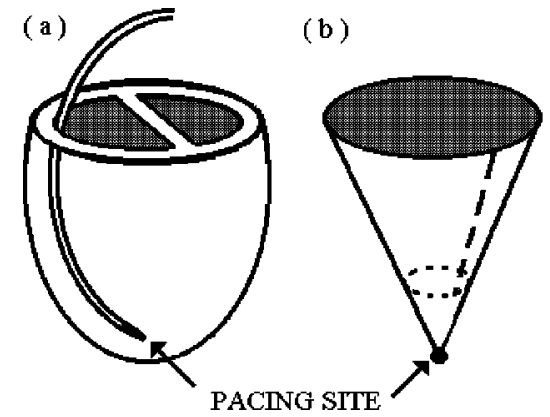

(c)

FIG. 5. (a) Schematic diagram of a typical cardiac pacing arrangement with an electrode from the ICD attached to the endocardial surface of the right ventricle. The electrode (shaded black) is at the apex of the ventricle (pacing site). (b) The ventricles can be represented by a cone with the electrode from the ICD attached at the apex - the pacing site. The broken lines indicate the way the cone is "opened up" to form the $2 \mathrm{D}$ simulation domain shown in (c). The long dashes in (b) represent the line along which the cone is cut to "open" it-the corresponding vertical edges in the 2D representation therefore have periodic boundary conditions. The line formed by the short dashes truncate the cone a fixed distance above the apex. The region below this line (including the pacing site) is not explicitly included in the 2D plane shown in (c) - propagation through this region could be simply accounted for via a fixed delay time.

standard five-point difference stencil for the Laplacian. ${ }^{27}$ The spatial grid consists of a square lattice with $N \times N$ points; in our studies we have used values of $N$ ranging from 128 to 256. The time step is $\delta t=0.022$ dimensionless units. We define dimensioned time $T$ to be $5 \mathrm{~ms}$ and 1 spatial unit to be $1 \mathrm{~mm}$. The scaled diffusion constant for the homogeneous system is $2 \mathrm{~cm}^{2} / \mathrm{s}$.

For the top and bottom of the 2D simulation domain we use no-flux (Neumann) boundary conditions because the ventricles are electrically insulated from the atria (top) and the waves converge and annihilate at the region around the apex (bottom) [Fig. 5(c)]. For the sides, we use periodic boundary conditions to represent the "wraparound" nature of the ventricle. Two rectangular patches of an inexcitable region (with diffusion constant $D=0$ ) are placed about the center of the simulation domain with a narrow passage for wave propagation between them. No-flux boundary conditions are used at the interface between the active medium and the inexcitable obstacle. We use this arrangement to represent anatomical obstacles (e.g., scar tissue) around which "figure-of-eight" reentry can occur, as depicted schematically in Fig. 1.

Our initial condition is a wavefront initiated at some point in the narrow conducting channel between the two inexcitable regions. A conduction block immediately below the point of initiation (implemented by making the corresponding region refractory) permits the propagation of the wave along a single direction (upward) only. The wave proceeds along the narrow channel and then around the two anatomical obstacles, setting up a figure-of-eight reentry pattern.

We looked at the effectiveness of different pacing algorithms in terminating figure-of-eight reentry. The pacing was simulated by a planar wave initiated at the base of the simulation domain. This represents a wave that has propagated a fixed distance up the ventricular wall from a local electrode at the apex-similar to a wave initiated at the tip of an in- 

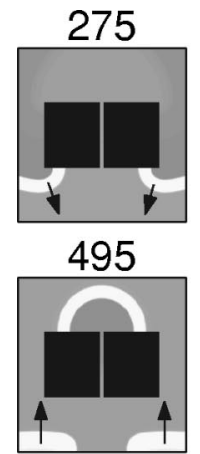

715

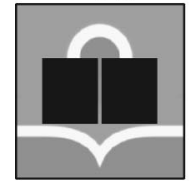

935

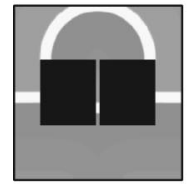

1155

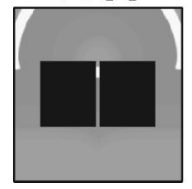

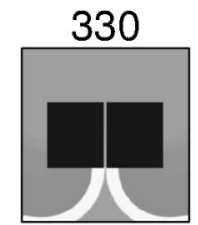

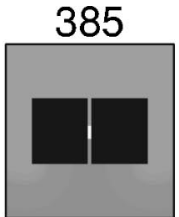

550

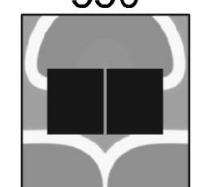

770

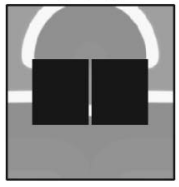

990

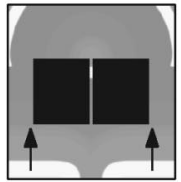

1210

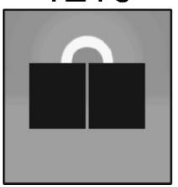

605

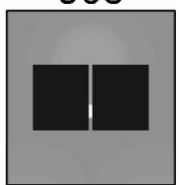

825

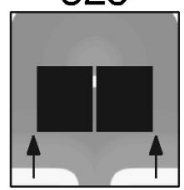

1045

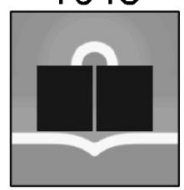

1265

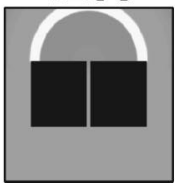

440

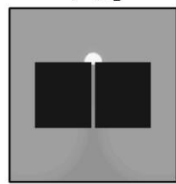

660

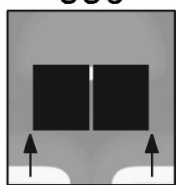

880

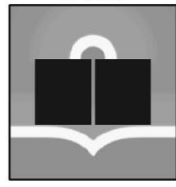

1100

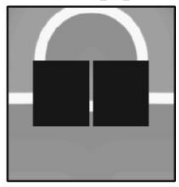

1320

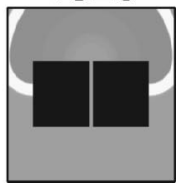

FIG. 6. Snapshots of waves (in white) at different times (labeled in msec above each plot) for the excitability $e$, upon application of a 4-stimulus pacing burst (applied at $462 \mathrm{~ms}, 627 \mathrm{~ms}, 792 \mathrm{~ms}$, and $957 \mathrm{~ms}$ ). Upon first appearance of each wave, its direction is indicated by a black arrow. The initial condition was a wave rotating around the rectangular obstacles (in black) as seen in the first four panels. The simulation domain $(128 \mathrm{~mm}$ $\times 128 \mathrm{~mm})$ is completely homogeneous with a diffusion constant $D$ $=2 \mathrm{~cm}^{2} / \mathrm{s}$. The two inexcitable obstacles are each $48.5 \mathrm{~mm}$ long and $43 \mathrm{~mm}$ wide, while the channel between the two obstacles has a width of $2.5 \mathrm{~mm}$. Pacing failed to terminate the reentrant wave.

verted cone before propagating up its walls [Fig. 5(b)].

Two different pacing patterns were applied. The first used pacing bursts at a cycle length which was a fixed percentage of the tachycardia cycle length (determined by the size of the obstacles). This corresponds to "burst pacing" used in ICDs. ${ }^{28}$ The pacing cycle length as well as the number of extrastimuli in each pacing burst (4-8) were varied to look at their effects on the reentrant wave. The second was decremental ramp pacing in which the interval between successive extrastimuli was gradually reduced [corresponding to "ramp pacing" used in ICDs (Ref. 28).]

For a homogeneous cardiac medium, both ramp and burst pacing were unsuccessful at terminating reentry. An example of the time evolution of such a procedure with a 4-stimulus pacing burst is shown in Fig. 6. We tried up to 8 -stimulus pacing trains and a comprehensive range of combinations of coupling and pacing intervals, all without successful termination. The reason for the failure of pacing to terminate reentry is similar to that in the 1D model. As the pacing wave approaches the reentry circuit, it is intercepted by the outwardly propagating wavefront originating from the

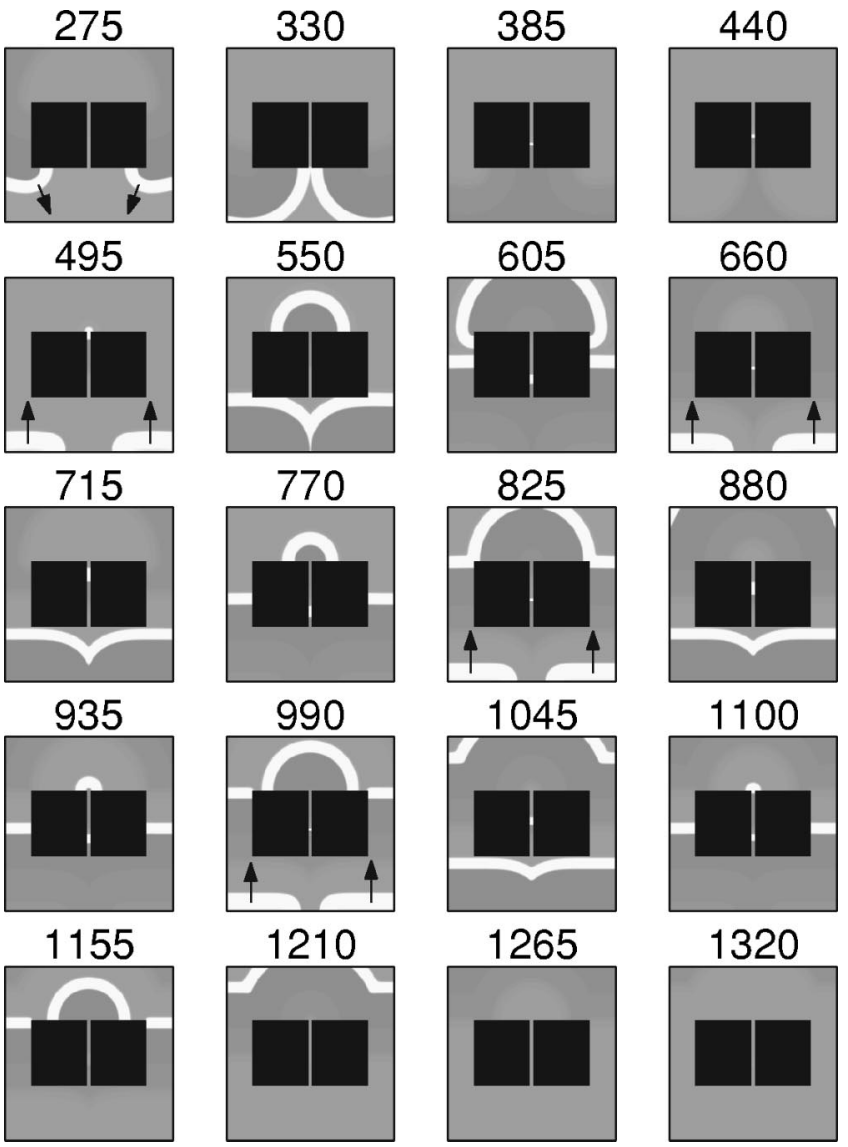

FIG. 7. Snapshots of waves at different times (labeled in msec above each plot) for the excitability $e$, upon application of a 4-stimulus pacing burst (applied at $462 \mathrm{~ms}, 627 \mathrm{~ms}, 792 \mathrm{~ms}$, and $957 \mathrm{~ms}$ ). The simulation domain has a region of slow conduction in the narrow channel between the two inexcitable obstacles, of length $7.5 \mathrm{~mm}$ and a diffusion constant $D^{\prime}$ $=0.1 \mathrm{~cm}^{2} / \mathrm{s}$. The fourth stimulus is blocked at the boundary of the inhomogeneity, leading to successful termination by $t \sim 1250 \mathrm{~ms}$.

reentry circuit, resulting in mutual annihilation (not shown). During the ensuing refractory period a pacing wave cannot approach the circuit at all. If a stimulus is applied immediately after the end of the refractory period, the wave propagates into the simulation domain (see the panels corresponding to $t=495-550 \mathrm{~ms}$ in Fig. 6), mutually annihilating with the reentrant wave at some point in the circuit (as occurs in the regions between the anatomical barriers and the vertical domain boundaries in the time between the $t=550 \mathrm{~ms}$ and $605 \mathrm{~ms}$ snapshots of Fig. 6). However, the branch of the stimulated wave proceeding along the narrow passage between the inexcitable obstacles continues on its path (see the panel corresponding to $t=605 \mathrm{~ms}$ in Fig. 6). When stimulation is halted, this intrachannel impulse gives rise again to a reentrant wave (e.g., the panels corresponding to $t$ $=1155-1320 \mathrm{~ms}$ in Fig. 6). This merely resets the reentry rather than terminating it. Only by blocking the branch of the stimulus propagating along the narrow channel is it possible to achieve successful termination.

As shown in Fig. 7, termination via such intrachannel blocking is possible in the presence of inhomogeneity. This is accomplished by placing a small zone (7.5 $\mathrm{mm}$ in length) of slow conduction in the narrow channel between the two 

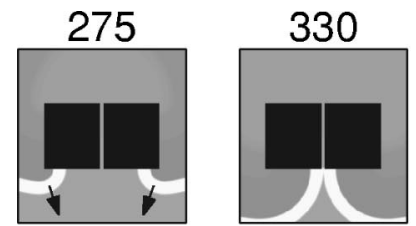

495

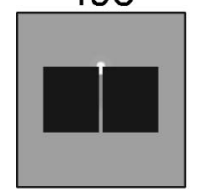

715

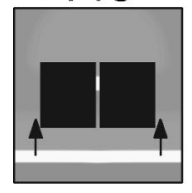

935

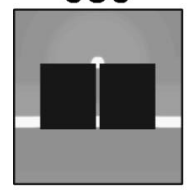

1155

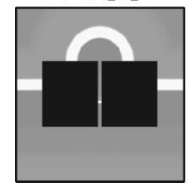

550

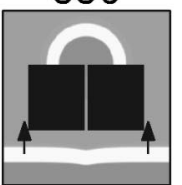

770

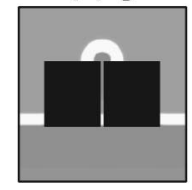

990

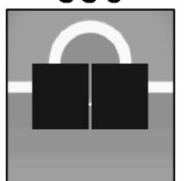

1210

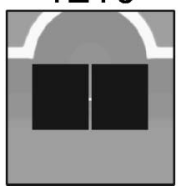

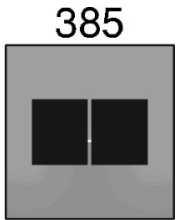

FIG. 8. Snapshots of waves at different times (labeled in msec above each plot) for the excitability $e$, upon application of a 4 -stimulus pacing burst (applied at $495 \mathrm{~ms}, 660 \mathrm{~ms}, 825 \mathrm{~ms}$, and $990 \mathrm{~ms}$ ). The simulation domain has a region of slow conduction in the narrow channel between the two inexcitable obstacles, of length $7.5 \mathrm{~mm}$ and a diffusion constant $D^{\prime}$ $=0.1 \mathrm{~cm}^{2} / \mathrm{s}$. The distinction from Fig. 7 is in the relative timing of the stimulus with respect to the reentrant wave, which results in failure of termination. The pacing interval $(=165 \mathrm{~ms})$ is the same in both figures. Pacing failed to terminate the reentrant wave.

nonconducting patches. The diffusion constant in this region was 0.05 times smaller than the rest of the tissue. A 4-stimulus pacing burst (with a pacing interval of $165 \mathrm{~ms}$ ) was found to successfully block the anterograde branch of the extrastimulus traveling through the narrow channel, and hence terminate the reentry (see the panels corresponding to $t=1155-1320 \mathrm{~ms}$ in Fig. 7). The timing of the stimulus relative to the reentrant wave is important, as applying the pacing at a slightly later time (but with same pacing interval) leads to failure of termination (Fig. 8).

Cardiac tissue also exhibits anisotropy-the action potential propagates faster along the direction of the myocardial fibers (longitudinal) than perpendicular to it (transverse). Although this is a type of inhomogeneity, the spatial gradient of the diffusion constant is much more gradual than that of the inhomogeneous region we have used in our simulation shown in Fig. 7. As we have seen in Sec. III, with onedimensional models, the parameter space in which successful termination can occur decreases with the increasing smoothness of the transition at the boundary of the inhomogeneity. This implies that there is a critical spatial gradient of intercellular resistance below which the inhomogeneity will not

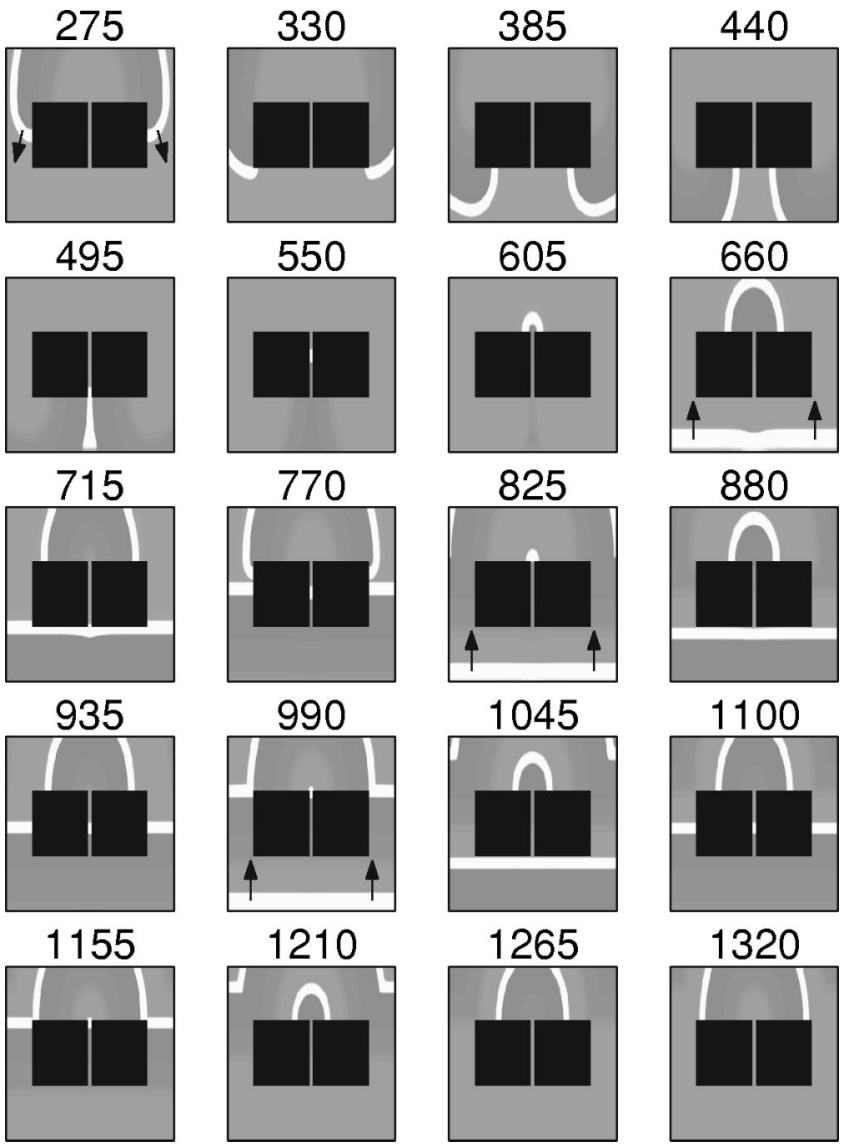

FIG. 9. Snapshots of waves at different times (labeled in ms above each plot) for the excitability $e$, upon application of a 4-stimulus pacing burst (applied at $462 \mathrm{~ms}, 627 \mathrm{~ms}, 792 \mathrm{~ms}$, and $957 \mathrm{~ms}$ ). The simulation domain has an anisotropy ratio of 1:0.3 for the diffusion constants, with the fast axis oriented along the up-down direction. Pacing failed to terminate the reentrant wave.

block the anterograde branch of the applied stimulus. Because anisotropy is a very gradual inhomogeneity, anterograde wave propagation is not disrupted enough by the anisotropy to lead to block and termination of propagation. This conclusion is supported by our 2D simulation, in which we looked at the effect of anisotropy by making the conductivities along the vertical and the horizontal axes differ in a ratio of 1:0.3, which is consistent with cardiac tissue. ${ }^{29}$ As with the homogeneous case, in the anisotropic case, none of the pacing methods we tried were successful in terminating reentry (one example is shown in Fig. 9).

For the 2D studies, in addition to the Panfilov model, we used two other 2-variable modified Fitzhugh-Nagumo-type models: the Aliev-Panfilov model (parameters are those given in Ref. 30), which simulates the restitution property of cardiac tissue, as well as the model proposed by Kogan et al. (parameters are those given in Ref. 31), which simulates the restitution and dispersion properties of cardiac tissue. In both cases the results were qualitatively similar to that obtained in the case of the Panfilov model and support the model independence of the $2 \mathrm{D}$ results.

\section{CONCLUSION}

There are certain limitations of our study. We have looked at one and two-dimensional models of anatomical 
reentry. However, the heart's three-dimensional structure is important in reentry, as the rotation of the axis of anisotropy along the thickness of the myocardium governs the propagation of impulses along the ventricle. We have also assumed the heart to be a monodomain rather than a bidomain (which has separate equations for intracellular and extracellular space). We believe this simplification to be justified for the low antitachycardia pacing stimulus amplitude. We have used a higher degree of cellular uncoupling to simulate ischemic tissue where slow conduction occurs. However, there are other ways of simulating ischemia, ${ }^{32}$ including, by increasing the external $\mathrm{K}^{+}$ion concentration. ${ }^{33}$ But this is transient and does not provide a chronic substrate for inducing arrhythmias.

Despite these limitations, the results presented here offer insight into pacing termination of anatomical reentry in the ventricle. We have developed model-independent mathematical arguments, supported by one- and two-dimensional simulations, that under certain conditions, the presence of circuit inhomogeneities is required for termination of anatomicalreentry VT through an unidirectional block like mechanism when stimulation occurs off the circuit (as is typical in reality). Given the clear dynamical similarity between the bidirectional block required for reentry termination and the unidirectional block required for reentry initiation ${ }^{4}$ it is noteworthy that the inhomogeneity-mediated mechanism of termination suggested here is consistent with the widely accepted theory that initiation of reentry is facilitated by circuit inhomogeneities $^{4,34}$ (but does not necessarily require a zone of slow conduction, in particular ${ }^{35}$ ). Considering the crucial role of such inhomogeneities in future studies may lead to more effective tachycardia termination pacing algorithms.

\section{ACKNOWLEDGMENTS}

We thank Bruce B. Lerman for helpful discussions. This work was supported by the American Heart Association (No. 0030028N).

\footnotetext{
${ }^{1}$ American Heart Association, 2001 Heart and Stroke Statistical Update (AHA, Dallas, 2001).

${ }^{2}$ Z.-J. Zheng, J. B. Croft, W. H. Giles, and G. A. Mensah, Circulation 104, 2158 (2001).

${ }^{3}$ J. A. Abildskov and R. L. Lux, J. Electrocardiol. 28, 107 (1995).

${ }^{4}$ Y. Rudy, J. Cardiovasc. Electrophysiol. 6, 294 (1995).
}

${ }^{5}$ J. M. Davidenko, R. Salomonsz, A. M. Pertsov, W. T. Baxter, and J. Jalife, Circ. Res. 77, 1166 (1995).

${ }^{6}$ M. E. Josephson, Clinical Cardiac Electrophysiology: Techniques and Interpretation, 2nd ed. (Lea Febiger, Philadelphia, 1993).

${ }^{7}$ H. Fei, M. S. Hanna, and L. H. Frame, Circulation 94, 2268 (1996).

${ }^{8}$ W. Quan and Y. Rudy, PACE 14, 1700 (1991).

${ }^{9}$ L. Glass and M. E. Josephson, Phys. Rev. Lett. 75, 2059 (1995).

${ }^{10}$ T. Gedeon and L. Glass, in Fields Institute Communications: Differential Equations with Applications to Biology (American Mathematical Society, Providence, RI, 1998), pp. 225-236.

${ }^{11}$ T. Nomura and L. Glass, Phys. Rev. E 53, 6353 (1996).

${ }^{12}$ L. Glass, Y. Nagai, K. Hall, M. Talajic, and S. Nattel, Phys. Rev. E 65, 021908 (2002).

${ }^{13}$ P. Comtois and A. Vinet, Chaos 12, 903-922 (2002).

${ }^{14}$ S. Sinha and D. J. Christini, e-print nlin.CD/0207061.

${ }^{15}$ N. El-Sherif, A. Smith, and K. Evans, Circ. Res. 49, 255 (1981).

${ }^{16}$ M. E. Rosenthal and M. E. Josephson, Circulation 82, 1889 (1990).

${ }^{17}$ W. G. Stevenson, H. Khan, P. Sager, L. A. Saxon, H. R. Middlekauff, P. D. Natterson, and I. Wiener, Circulation 88, 1647 (1993); W. G. Stevenson, P. T. Sager, and P. L. Friedman, J. Cardiovasc, J. Cardiovasc. Electrophysiol. 6, 201 (1995); K. Soejima, W. G. Stevenson, W. H. Maisel, E. Delacretaz, C. B. Brunckhorst, K. E. Ellison, and P. L. Friedman, J. Am. Coll. Cardiol. 37, 1386 (2001).

${ }^{18}$ C. de Chillou, D. Lacroix, D. Klug, I. Magnin-Poull, C. Marqui, M. Messier, M. Andronache, C. Kouakam, N. Sadoul, J. Chen, E. Aliot, and S. Kacet, Circulation 105, 726 (2002).

${ }^{19}$ C. Ingelmo and L. H. Frame, J. Cardiovasc. Electrophysiol. 11, 981 (2000).

${ }^{20}$ C. H. Luo and Y. Rudy, Circ. Res. 68, 1501 (1991).

${ }^{21}$ A. G. Kleber, C. B. Riegger, and M. J. Janse, Circ. Res. 51, 271 (1987).

${ }^{22}$ W. Quan and Y. Rudy, Circ. Res. 66, 367 (1990).

${ }^{23}$ R. W. Joyner, Biophys. J. 35, 113 (1981); R. W. Joyner, R. Veenstra, D. Rawling, and A. Chorro, ibid. 45, 1017 (1984).

${ }^{24}$ A. V. Panfilov and P. Hogeweg, Phys. Lett. A 176, 295 (1993); A. V. Panfilov, Chaos 8, 57 (1998).

${ }^{25}$ S. Sinha, A. Pande, and R. Pandit, Phys. Rev. Lett. 86, 3678 (2001).

${ }^{26}$ A. V. Panfilov and J. P. Keener, J. Theor. Biol. 163, 439 (1993).

${ }^{27}$ W. H. Press, S. A. Teukolsky, W. T. Vetterling, and B. P. Flannery, Numerical Recipes in $C$ (Cambridge University Press, London, 1995)

${ }^{28}$ L. Horwood, S. VanRiper, and T. Davidson, Am. J. Crit. Care 4, 397 (1995).

${ }^{29}$ J. P. Keener and A. V. Panfilov, in Computational Biology of the Heart, edited by A. V. Panfilov and A. V. Holden (Wiley, New York, 1997), p. 235.

${ }^{30}$ R. R. Aliev and A. V. Panfilov, Chaos, Solitons Fractals 7, 293 (1996).

${ }^{31}$ B. Y. Kogan, W. J. Karplus, B. S. Billett, A. T. Pang, H. S. Karagueuzian, and S. S. Khan, Physica D 50, 327 (1991).

${ }^{32}$ R. M. Shaw and Y. Rudy, Circ. Res. 80, 124 (1987).

${ }^{33}$ F. Xie, Z. Qu, A. Garfinkel, and J. N. Weiss, Am. J. Physiol. Heart Circ. Physiol. 280, H1667 (2001).

${ }^{34}$ H. Zhang, R. W. Winslow, and A. V. Holden, J. Theor. Biol. 191, 279 (1998).

${ }^{35}$ S. D. Girouard and D. S. Rosenbaum, J. Cardiovasc. Electrophysiol. 12, 697 (2001). 